\title{
Deformation-induced phase transformation in zinc sulphide
}

\author{
L. A. XUE, ${ }^{*}$ R. RAJ \\ Department of Materials Science and Engineering, Cornell University, Ithaca, New York 14853-1501, \\ USA
}

There are two crystalline modifications of zinc sulphide. The high-temperature hexagonal $(\alpha)$ phase and the low-temperature cubic $(\beta)$ phase exhibit a reconstructive transformation at a transition temperature of about $1020^{\circ} \mathrm{C}$. The transformation of $\alpha$ phase back to $\beta$ phase is a sluggish process compared to that of $\beta$ to $\alpha$ [1], therefore once it is formed the $\alpha$ phase will remain as a metastable phase at room temperature. The presence of $\alpha-\mathrm{ZnS}$ in a $\beta$ - ZnS matrix produces a twophase material; this is detrimental to the optical properties because of the resultant scattering loss for transmitted light due to a small mismatch in the refractive indices of the two $\mathrm{ZnS}$ phases [2].

It has been shown that the transformation of a metastable hexagonal phase containing $\mathrm{ZnS}$ single crystal to a complete cubic structure can be achieved through plastic deformation at room temperature by applying shear stress along a (1 111$)$ slip plane [3]. In this letter we report our observation of deformationdependent phase transformation in polycrystalline $\mathrm{ZnS}$ near its phase transition temperature.

$\mathrm{ZnS}$ pellets were prepared by hot-pressing from a high-purity $\mathrm{ZnS}$ powder (G.E. $>99.9 \%$ ) under pressure of $200 \mathrm{MPa}$ and temperature range of 900 to $1050^{\circ} \mathrm{C}$ for a period of 15 to $90 \mathrm{~min}$. The temperature and time varied to control the $\alpha$ phase content. Some of the starting $\mathrm{ZnS}$ discs were obtained from Eastman Kodak Co. Specimens for the deformation experiments were cut as rectangular parallelepipeds of approximate size $4.5 \mathrm{~mm} \times 4.5 \mathrm{~mm} \times 6 \mathrm{~mm}$ or $7 \mathrm{~mm} \times$ $7 \mathrm{~mm} \times 9 \mathrm{~mm}$. The $\alpha$ phase content of the starting material ranged from 2.9 to $43.3 \%$. The testing was done in uniaxial compression at constant displacement rates, in argon at one atmosphere pressure. The displacement rates used were from 0.01 to $1 \mathrm{~mm}$ $\mathrm{min}^{-1}$, corresponding to initial strain rates of $1.4 \times$ $10^{-5}$ to $2 \times 10^{-3} \mathrm{sec}^{-1}$. X-ray diffraction was employed to determine the $\alpha$-ZnS contents of the samples before and after deformation testing, using the procedure described elsewhere [4]. These results were then compared with those obtained from static annealing of samples under otherwise identical conditions.

Fig. 1 shows the $\alpha$-ZnS content as a function of deformation or annealing time at $1000^{\circ} \mathrm{C}$ for various initial $\alpha$ contents. It is clear that at this temperature deformation accelerates the $\beta$ to $\alpha$ phase transition in the whole $\alpha$-content range studied. From the figure, it would seem that the transformation is strongly strainrate or flow-stress dependent. This is not really the case. In Fig. 2 we plot data in the form of $\Delta \alpha$ (difference of $\alpha$ content between deformed samples and annealed ones at a given time) against the strain for samples having initial $\alpha$ contents of 2.9 and $5.5 \%$. There is a nearly linear relationship of $\Delta \alpha$ and the strain. The same trend was observed at other temperatures in the cubic $\mathrm{ZnS}$ range. For example, results obtained at $950^{\circ} \mathrm{C}$ give a similar strain dependence of the transformation, as shown in Fig. 3. Such a correlation between the strain and $\Delta \alpha$ during the dynamic phase transformation of polycrystalline $\mathrm{ZnS}$ is consistent with the findings in single-crystal $\mathrm{ZnS}$ deformed at room temperature [3]. This suggests that the conversion of metastable hexagonal $\mathrm{ZnS}$ structure to the cubic one may result from successive glide of partial dislocations creating the same shear in each of the slip planes. In polycrystalline $\mathrm{ZnS}$, however, due to co-existence of the dislocation and diffusional

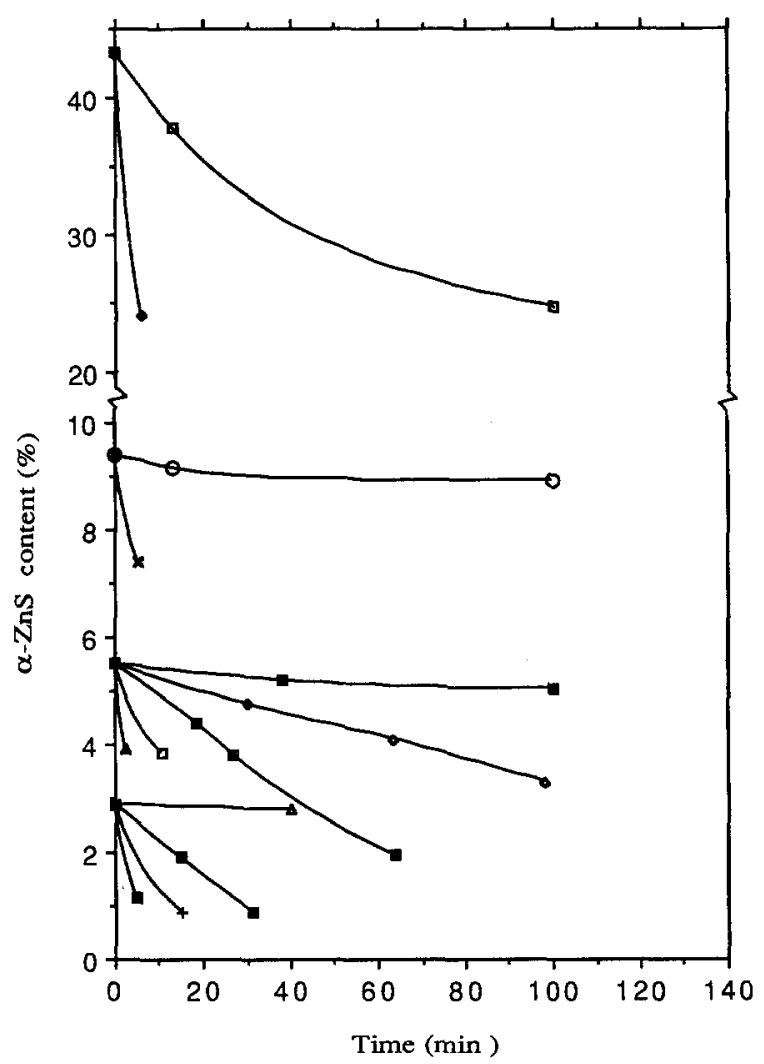

Figure $l \alpha-\mathrm{ZnS}$ content as a function of deformation/annealing time at $1000^{\circ} \mathrm{C}$ for various initial $\alpha$ contents. Strain rates $\left(\mathrm{sec}^{-1}\right)$ as follows from top to bottom: (E) $0,(\diamond) 3.3 \times 10^{-4}$; (O) 0 , (x) $3.4 \times 10^{-4} ;$ (घ) 0, (৫) $7.0 \times 10^{-5}$, (口) $1.9 \times 10^{-4}$, () $7.0 \times 10^{-4}$, (ム) $2.0 \times 10^{-3} ;$ (A) 0 , (ロ) $3.6 \times 10^{-4}$, (+) $7.4 \times 10^{-4},(\mathbf{a}) 1.8 \times 10^{-3}$. 


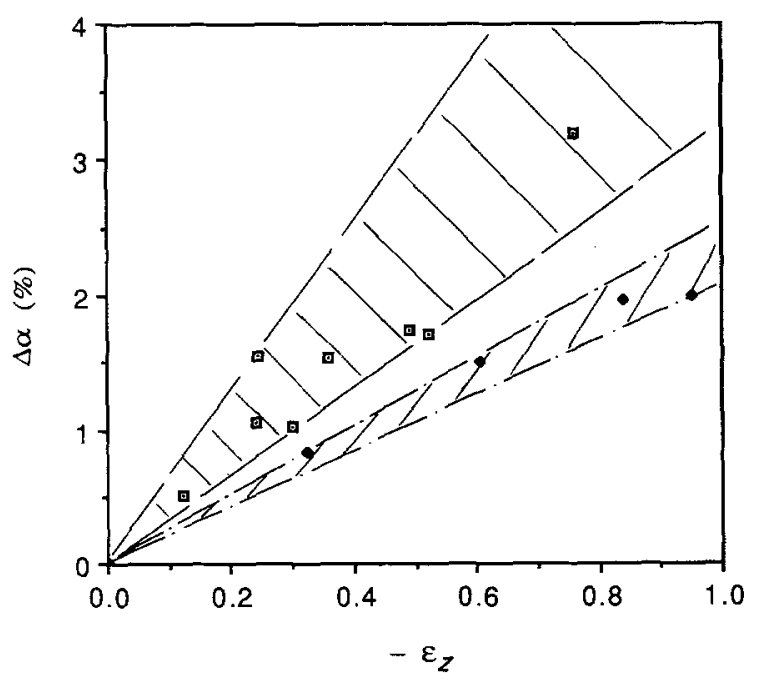

Figure $2 \Delta \alpha$ against strain data at $1000^{\circ} \mathrm{C} ; \alpha_{0}=(\boxminus) 5.5 \%$, (৮) $2.9 \%$,

mechanisms, and also due to the random distribution of crystal orientations of each grain, greater strain would be needed than in the case of a single crystal to achieve a certain amount of phase transformation.

The strain rate or flow stress, on the other hand, also seems to have some positive influence on the $\alpha$ to $\beta$ transformation. In general a higher strain rate, i.e. higher flow stress, gives rise to a slightly higher $\Delta \alpha$, as can be seen from Fig. 2. This partial flow-stress dependence of the transformation may be interpreted as the favoured effect of the compression pressure during the $\alpha$ to $\beta$ phase transformation which undergoes a $3 \%$ volume reduction.

The effect of strain and flow stress on the reverse process, i.e. $\beta$ to $\alpha$ transformation, is less unambiguous. It has been shown [4] that at $1050^{\circ} \mathrm{C}$, which is just above the transition temperature, the transformation is correlated not with the strain, but with the

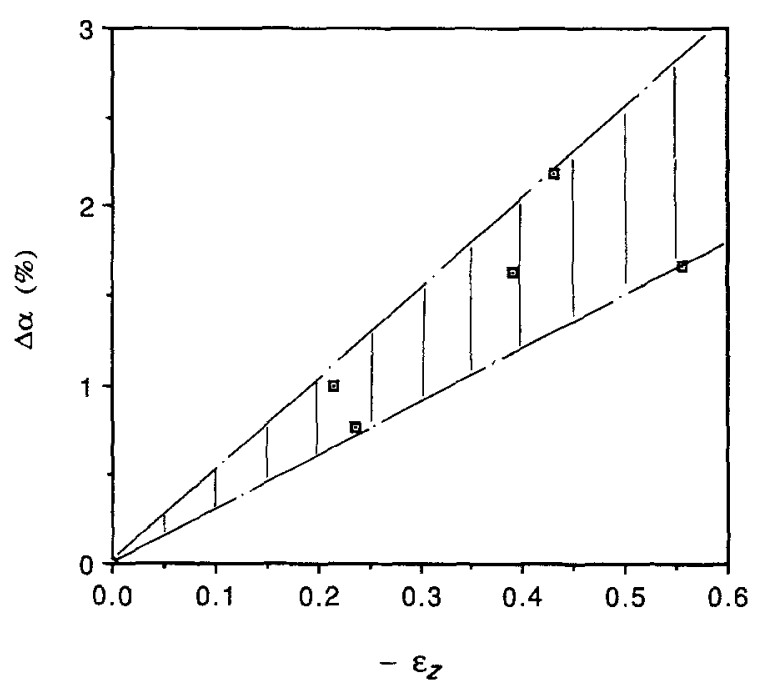

Figure $3 \Delta \alpha$ against strain data at $950^{\circ} \mathrm{C} ; \alpha_{0}=5.5 \%$.

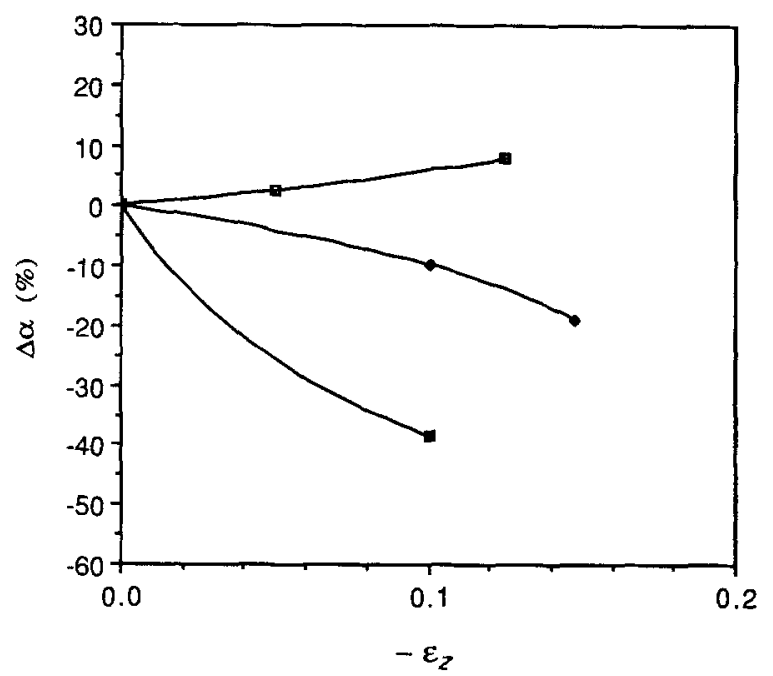

Figure $4 \Delta \alpha$ against strain data at $1150^{\circ} \mathrm{C}$ for various initial strain rates. $\alpha_{0}=5.5 \% ; \quad \dot{\varepsilon}=$ (ए) $1.5 \times 10^{-3}, \quad$ (৫) $4.0 \times 10^{-4}$, (a) $1.0 \times 10^{-4}$.

strain rate or flow stress, possibly through the favoured influence of the stress in the formation of twin planes [4]. The transformation, however, involves a volume increase of more than $3 \%$, therefore pressure can also have some negative effect on $\beta$ to $\alpha$ phase transformation. At even higher temperatures, for instance $1150^{\circ} \mathrm{C}$, while a high strain rate still appears to favour the transformation, $\Delta \alpha$ decreases with increase of strain at moderate strain rates as shown in Fig. 4.

To summarize, deformation-induced phase transformation in polycrystalline $\mathrm{ZnS}$ is observed in the temperature range 900 to $1150^{\circ} \mathrm{C}$. Below the cubic to hexagonal transition temperature of $\sim 1020^{\circ} \mathrm{C}$, the transformation of hexagonal to cubic structure is found to be strain-dependent. Above the transition temperature, high strain rate or flow stress appears to promote the cubic to hexagonal phase transformation.

\section{Acknowledgements}

This research was supported by the Office of Naval Research. Support was also received from the National Science Foundation through the use of the facilities of the Materials Science Center at Cornell University.

\section{References}

1. M. SAKAGUChI and T. HiRABAYAShi, J. Appl. Phys. 44 (1973) 2530.

2. L. A. XUE, D. S. FARQUHAR, T. W. NOH, A. J. SIEVERS and R. RAJ, Acta Materialia in press.

3. A. F. GUROV, M. P. KULAKOV, V. M. POLOVOV and S. Z. ShMUrak, Sov. Phys. Solid State 18 (1976) 2160.

4. L. A. XUE and R. RAJ, J. Amer. Ceram. Soc. 72 (1989) 1792.

Received 30 October

and accepted 14 November 1989 